\title{
FutureJournal
}

\section{Práticas de Recursos Humanos que Influenciam na Criatividade dos Funcionários}

\author{
Marjulie Merini Alberton \\ Universidade Regional de Blumenau (FURB), Brasil \\ marjuliealberton@gmail.com \\ Luciano Castro de Carvalho \\ Universidade Regional de Blumenau (FURB), Brasil \\ luccar@gmail.com
}

\section{RESUMO}

O objetivo neste estudo é descrever as práticas de recursos humanos que influenciam a criatividade dos funcionários. Para isso, optou-se por um estudo quantitativo, descritivo, de corte transversal. Foram pesquisados 119 funcionários de empresas diversas por meio de um questionário eletrônico contendo seis dimensões de análise: criatividade (variável dependente), autonomia, avaliação de desempenho, recompensa, participação e treinamento (variáveis independentes). Por meio da modelagem por equações estruturais, testou-se a relação das variáveis independentes com a dependente e verificou-se que autonomia, avaliação de desempenho, recompensa e participação exercem influência positiva e significante sobre a criatividade dos funcionários. Não foi encontrada relação significante entre treinamento e criatividade devido ao foco do treinamento para o exercício das atividades ser relacionado à função e não à geração de ideias.

PALAVRAS-CHAVE: Criatividade. Gestão de pessoas. Inovação. 


\title{
FutureJournal
}

\section{Human Resource Practices Influencing Employee Creativity}

\begin{abstract}
This study aims to describe the human resources practices that influence the employee creativity. To obtain this, the quantitative, descriptive and cross section research were used with 119 employees belonging to several companies and industries. It was used an online survey, encompassing six dimensions: creativity (dependent variable), autonomy, performance assessment, reward system, participation and training (independent variables). By structural equation modeling the relationship between independent variables and dependent variable was verified and the results have shown that the autonomy, performance assessment, reward system and participation have significant and positive influence on employees' creativity. It was not found any significant relation between training and creativity, once the focus of training was enabling the employee to execute the activity instead of generating ideas.
\end{abstract}

KEY-WORDS: Creativity. Human resources. Innovation. 


\section{INTRODUÇÃO}

A inovação torna-se relevante ao mercado dinâmico de negócios, que exige a introdução contínua de novos produtos e serviços, com o propósito de manter as empresas competitivas em âmbito mundial e garantir-Ihes a sobrevivência. Para as empresas inovarem, é necessário que os funcionários que nela atuam sejam criativos o bastante para contribuírem com novas ideias. De acordo com Amabile, Conti, Coon, Lazenby e Herron (1996), toda inovação começa com ideias criativas. Criatividade é a ideia inicial que conduz à inovação organizacional. Funcionários criativos contribuem com o processo inovativo e tornam-se recursos valiosos para as empresas.

Indivíduos criativos são mais dispostos, ousam e arriscam-se em atividades complexas. Jambulingam, Kathuria e Doucette (2005) afirmam que a vontade de assumir riscos contribui para o desejo da organização de desenvolver e implantar novas ideias. O pensar diferente, arriscar-se, superar obstáculos são características de perfis criativos que as empresas precisam para competir no mercado.

Para potencializar um ambiente propício à criatividade dos funcionários, a área de gestão de pessoas pode desenvolver práticas que estimulem a criatividade dos funcionários. As práticas de recursos humanos abrangem atividades e formalidades organizacionais, como recrutamento e seleção de pessoal, treinamento e desenvolvimento, avaliação de desempenho, motivação e retenção de funcionários. Conforme Binyamin e Carmeli (2010), organizações podem desempenhar papéis proativos, assim as práticas de recursos humanos podem ser estrategicamente aplicadas de maneira a fomentar a criatividade dos funcionários, criando ambientes de trabalho agradáveis que proporcionem autonomia e acesso facilitado aos recursos necessários, além de amparo da gestão transformacional e ética.

Embora haja uma relação lógica estabelecida entre práticas de gestão de pessoas e estímulo à criatividade para inovação, não foram encontrados estudos que tenham testado essa relação empiricamente. Em geral, estudos existentes têm relacionado as práticas de gestão de pessoas com desempenho organizacional (Zehir, Gurol, Karaboga \& Kole, 2016), desempenho emocional dos funcionários e serviço ao cliente (Gabriel, 
Cheshin, Moran \& van Kleef, 2016), inovações organizacionais e capacidade de gestão do conhecimento (Afacan Fındıklı, Yozgat \& Rofcanin, 2015), turnover (Slavich, Cappetta \& Giangreco, 2014), satisfação no trabalho (Paşaoğlu \& Tonus, 2014), desempenho dos funcionários(Nadarajah, Kadiresan, Kumar, Kamil \& Yusoff, 2012), má conduta dos funcionários (Werbel \& Balkin, 2010), comprometimento dos funcionários (Rogg, Schmidt, Shull \& Schmitt, 2001) e atração e retenção de talentos (Hiltrop, 1999).

Considerando que o desempenho do funcionário nas mais diversas áreas organizacionais depende da criatividade para inovar ou solucionar problemas, sugere-se neste estudo que as práticas de gestão de pessoas podem estimular a criatividade para a inovação. Dessa forma, surge o questionamento: Quais práticas de gestão de pessoas influenciam a criatividade dos funcionários? Neste estudo, visa-se fornecer às organizações esclarecimentos sobre os fatores pessoais e organizacionais que favorecem a criatividade dos funcionários, por meio das práticas de gestão de pessoas.

Para maior elucidação do tema de pesquisa, este artigo está subdividido em seis tópicos. O primeiro refere-se à Criatividade para Inovação e traz alguns conceitos sobre os temas. Aborda também fatores pessoais e organizacionais que podem impactar na criatividade das pessoas. O segundo tópico abrange o papel das pessoas no processo de inovação das empresas. Descreve os meios de trabalho que propiciam o desenvolvimento da inovação e como as pessoas podem contribuir positivamente na inovação organizacional. O tópico três destaca a importância das práticas de recursos humanos no processo inovativo das empresas e descreve algumas práticas de $\mathrm{RH}$ voltadas ao desenvolvimento criativo dos funcionários. No tópico quatro, metodologia, descrevem-se os procedimentos utilizados para a análise dos resultados, descrita no tópico cinco. Por fim, o tópico seis aborda as considerações finais deste artigo, juntamente com as delimitações e limitações da pesquisa. 


\section{REFERENCIAL TEÓRICO}

Nesta seção serão esclarecidos os principais tópicos da pesquisa: criatividade para inovação, papel das pessoas no processo de inovação das organizações e práticas de recursos humanos que estimulam a criatividade.

\subsection{CRIATIVIDADE PARA INOVAÇÃO}

Diversos conceitos sobre a criatividade individual são encontrados na literatura, tornando complexa a tarefa de definir a criatividade (K. K. Chen, 2012; Mumford \& Gustafson, 1988; Udwadia, 1990). Alguns autores sugerem que a criatividade pode ser considerada um fenômeno multivariado que envolve vários atributos cognitivos e características de personalidade (Mumford \& Gustafson, 1988). Outros defendem a criatividade como resultado da formação de várias associações no cérebro que geram ideias úteis e interessantes (Binyamin \& Carmeli, 2010; Udwadia, 1990). Para este artigo, assume-se que a criatividade é a ideia inicial para a geração de novos produtos, processos ou serviços visando atingir da melhor maneira os objetivos da organização (Amabile, 1988; Amabile, Barsade, Mueller \& Staw, 2005; Nayak, Noida, \& Agarwal, 2011).

A inovação em uma organização não ocorre sem ideias criativas por parte dos indivíduos. As ideias são primeiramente necessárias para o posterior desenvolvimento e implementação na empresa (Amabile, 1998; Amabile et al., 1996). A criatividade individual é considerada o elemento mais básico e crítico no processo inovativo de uma empresa e essencial para seu crescimento (Bai, Lin \& Li, 2016; K. K. Chen, 2012; Udwadia, 1990).

Criatividade e inovação organizacional são sistemas diferenciados, porém fortemente relacionados (Amabile, 1988; Borghini, 2005; Nayak et al., 2011). De maneira simplista, para diferenciá-los, a criatividade pode ser definida como o primeiro passo para a inovação. É a produção de ideias benéficas a qualquer área. Já a inovação pode ser definida como a implementação dessas ideias criativas dentro de uma empresa, relevante para o sucesso no longo prazo. Diante desse contexto, a criatividade é a 
primeira condição para a inovação, mas não suficiente para tal (Amabile, 1997; 2013; Amabile et al., 1996).

Com o objetivo de explorar quais elementos básicos e individuais propiciam a resposta criativa das pessoas, surge inicialmente o modelo componencial de criatividade, formado por três elementos: habilidades relevantes de domínio, habilidades relevantes para a criatividade e motivação intrínseca de tarefa. As habilidades relevantes de domínio referem-se aos conhecimentos tácitos e explícitos de cada pessoa. As habilidades relevantes para a criatividade abrangem a personalidade, a maneira como cada indivíduo se comporta em relação aos problemas e às tarefas. Por fim, a motivação intrínseca de tarefa refere-se ao interesse e à satisfação para realização da atividade, conduzindo a resultados mais criativos para a organização (Amabile, 1988; 1997; 1998; 2013; Amabile et al., 1996).

Uma extensão posterior do modelo de criatividade englobou a inovação organizacional, apresentando os componentes psicológicos e sociais que influenciam as pessoas a produzirem de forma criativa. Assim, o modelo componencial da criatividade passou a ser conhecido como modelo componencial de criatividade e inovação organizacional, contemplando elementos organizacionais que abrangem o ambiente de trabalho e afetam os indíviduos para que a inovação aconteça (Amabile, 1988, 2013; Amabile et al., 1996).

Os elementos organizacionais inseridos no modelo componencial da criatividade e inovação organizacional são a motivação organizacional para inovar, recursos e práticas de gestão. A motivação organizacional para inovar é a orientação básica da organização para a inovação, o apoio para a criatividade e a inovação na organização. Os recursos envolvem tudo que a organização tem disponível para auxiliar no trabalho inovativo. As práticas de gestão abrangem liberdade ou autonomia para realização da tarefa, atividades desafiadoras e interessantes, especificação clara dos objetivos empresariais e formação de equipes de trabalho com habilidades diversificadas (Amabile, 1988; 2013; Amabile et al., 1996).

Todos os elementos individuais e organizaionais descritos buscam melhorar a resposta criativa das empresas para as exigências do mercado de negócios competitivo. As mudanças tecnológicas requerem das empresas 
maior agilidade no processo comunicativo, busca por novas ideias e experiências, visando à melhoria de seu produto, processo ou tecnologia, designado como inovação organizacional (Nayak et al., 2011; Tan \& Nasurdin, 2011; Udwadia, 1990).

Percebe-se a inovação organizacional como elemento relevante às organizações para competitividade e sobrevivência de seus negócios. Ferramentas utilizadas para promover a inovação organizacional referem-se às práticas de gestão eficaz de recursos humanos e gestão do conhecimento. As práticas de recursos humanos estão claramente associadas com a capacidade de gestão do conhecimento, que, por sua vez, tem uma consequência positiva sobre o desempenho da inovação. Evidencia-se que a capacidade de gestão do conhecimento desempenha um papel moderador por meio das práticas de recursos humanos e desempenho da inovação (Borghini, 2005; C. -J. Chen \& Huang, 2009; Tan \& Nasurdin, 2011).

Com o intuito de estimular os funcionários a adquirir, compartilhar e aplicar o conhecimento nas organizações, as empresas promovem práticas de recursos humanos. Práticas adequadas de recursos humanos podem apoiar e fomentar o desenvolvimento de um ambiente organizacional propício para as atividades de gestão do conhecimento. Logo, a perspectiva é de que as práticas de recursos humanos estimulam de foma positiva a capacidade de gestão do conhecimento (C. - J. Chen \& Huang, 2009).

\subsection{O PAPEL DAS PESSOAS NO PROCESSO DE INOVAÇÃO NAS EMPRESAS}

De maneira generalizada, as organizações identificam que parte da criatividade provém dos funcionários, o que é conhecido como capital humano ou intelectual. Assim, investir no capital humano das empresas é uma das bases para o sucesso organizacional (Nayak et al., 2011; Snell \& Dean, 1992). O capital intelectual é resultado do investimento de uma organização, seja sob a forma de contratação de determinado funcionário, seja pelo desenvolvimento interno deste (Snell \& Dean, 1992).

Os capitais intelectuais de uma organização são recursos valiosos para aquelas que almejam alcançar a inovação diferenciada e vantagens competitivas sustentáveis (C. -J. Chen \& Huang, 2009; Snell \& Dean, 1992). 
Diversos estudos têm procurado verificar os efeitos dos processos de recursos humanos como recrutamento, treinamento, avaliação e recompensação de resultados dos funcionários, de modo a manter condições propícias para a geração de criatividade (Binyamin \& Carmeli, 2010; Snell \& Dean, 1992).

As organizações necessitam gerenciar o capital humano por meio das variadas práticas de recursos humanos de modo a aumentar a capacidade de gestão do conhecimento, compartilhamento e aplicação. Melhores níveis da capacidade de gestão do conhecimento podem estimular pensamentos criativos e inovadores que, consequentemente, podem melhorar 0 desempenho da inovação (C. -J. Chen \& Huang, 2009).

Dessa forma, a criatividade depende de uma série de elementos, como os processos implícitos à capacidade pessoal para geração de novas ideias, características pessoais que facilitam o processo e a implementação das ideias, as particularidades da situação que mantêm a vontade do indivíduo de envolver-se num comportamento criativo e a avaliação do esforço produtivo do indivíduo (Mumford \& Gustafson, 1988). Observa-se que fatores internos e externos, podem estimular ou reduzir a criatividade. Os esforços para resolver esses dilemas podem afetar diretamente a criatividade (K. K. Chen, 2012).

Qualidades pessoais relacionadas com traços de personalidade como persistência, curiosidade, energia e honestidade podem ser cultivados para promover a criatividade individual (Agnoli \& Corazza, 2015). Indivíduos que acreditam ter energia para realizar atividades inovadoras tendem a ser mais dispostos e propensos a exercer as tarefas de trabalho criativo (Binyamin \& Carmeli, 2010). Percebe-se que funcionários tendem a produzir de maneira mais criativa quando apresentam características pessoais consideradas positivas e adequadas à criatividade, além de atuarem de forma mais segura nas tarefas desafiadoras em empregos complexos, amparados por líderes solidários e não controladores (Borghini, 2005).

Pessoas criativas tendem a solucionar problemas de maneiras diferentes, correndo riscos que os demais evitam, superando medos e desafiando a maioria para sustentar suas ideias, buscando transpor diversos obstáculos (Fischer, Oget \& Cavallucci, 2016). As novas ideias e as tentativas 
de implementação estão sujeitas a erros e riscos, porém esses erros levam à introdução de mudanças e inovações na organização, visto que os indivíduos buscam formas de corrigi-los ou experimentar outras ideias (K. K. Chen, 2012; Jambulingam et al., 2005). Perfis mais automotivados, com habilidades cognitivas desenvolvidas, tendem a ser mais criativos. Outra qualidade que influencia na promoção da criatividade é a experiencia que o indivíduo possui na área e a habilidade social, além das qualidades e experiências diversas do grupo de trabalho (Amabile, 1988; 1998; K. K. Chen, 2012).

As pessoas tornam-se mais criativas quando se sentem motivadas pela satisfação, pelo interesse, pelo prazer e pelo desafio que a atividade lhe proporciona (Amabile, 1997). Essa satifação na realização da atividade criativa, pode levar o indivíduo a entrar num ciclo virtuoso de criatividade (Amabile et al., 2005; Mumford \& Gustafson, 1988). A motivação interna do indivíduo tende a gerar mais ideias criativas do que as motivações externas por parte da organização (Amabile, 1988; 1997).

\subsection{PRÁTICAS DE GESTÃO DE PESSOAS NO PROCESSO INOVATIVO}

Em função da competitividade e da instabilidade do ambiente empresarial em âmbito mundial, as empresas têm buscado melhorar e diferenciar-se por meio das práticas de gestão de recursos humanos, adaptando-se constantemente ao mercado dinâmico (Binyamin \& Carmeli, 2010; C.-J. Chen \& Huang, 2009). As práticas de recursos humanos estão relacionadas com atividades específicas referentes às pessoas e às formalidades da organização, além de filosofias que são utilizadas para atrair, desenvolver, motivar e reter os funcionários que asseguram o funcionamento correto e a sobrevivência da empresa (Tan \& Nasurdin, 2011). Assim, as empresas devem avaliar as práticas de recursos humanos existentes e, se necessário, modificá-las, objetivando maior contribuição dos funcionários na melhoria do desempenho (Ahmad \& Schroeder, 2003).

Assimilar os fatores elementares que influenciam na capacidade da organização para novas ideias e inovação de seus produtos ou processos é uma tarefa estratégica relevante para sustentar a vantagem competitiva. 
Essa necessidade de competitividade para a sobrevivência impulsionou as empresas a compreenderem melhor a criatividade nas organizações e suas práticas de recursos humanos (Beugelsdijk, 2008; Tan \& Nasurdin, 2011).

Sugere-se que a estruturação adequada dos processos de gestão de recursos humanos pode fomentar a criatividade dos empregados (Binyamin \& Carmeli, 2010). As práticas de recursos humanos podem beneficiar a empresa com uma cultura e estrutura organizacional funcional com base em conhecimentos (C.-J. Chen \& Huang, 2009). As empresas, porém, devem equilibrar seus processos, cuidando do excesso de organização e burocracia, e encontrando maneiras de não limitar a criatividade. Processos exageradamente burocráticos tendem a inibir o processo criativo e, consequentemente, a inovação nas organizações (K. K. Chen, 2012). Fatores pessoais e organizacionais podem auxiliar na melhoria da criatividade nas organizações e, embora cada fator seja único, um complementa o outro e juntos somam para a melhoria da criatividade organizacional (Ahmad \& Schroeder, 2003; C.-J. Chen \& Huang, 2009; Liu, Combs, Ketchen Jr \& Ireland, 2007).

Para fins de compreensão do processo criativo nas organizações, deve-se considerar o conhecimento compatilhado entre os indivíduos, os modelos mentais e também os valores e cultura organizacional (Borghini, 2005). O processo criativo pode ser dificultado em culturas muito formais e tradicionais, assim como a liderança autoritária também pode ter efeito inibidor sobre o processo (A. S.-Y. Chen \& Hou, 2016; K. K. Chen, 2012).

Estudos apontam a relação entre a liderança transformacional e o nível de criatividade dos funcionários. Um breve conceito desse estilo de liderança descreve líderes inspiradores que conquistam seguidores por meio de seu carisma e comportamento exemplar, visando ampliar e elevar os interesses de seus seguidores, estimulando-os a superar desafios. Observouse que a motivação intrínseca dos indivíduos tende a aumentar conforme os líderes apresentam alta liderança transformacional. O contrário também ocorre: quando a liderança transformacional é baixa, o efeito indireto da variedade cognitiva na criatividade por meio da motivação intrínseca é negativa (Bai et al., 2016; Sarinf \& Dermott, 2003; X.-H. Wang, Kim \& Lee, 2016). 
Outros estudos fazem o levantamento da relação positiva entre a liderança ética e a criatividade. Sugere-se que, quando os funcionários percebem excelente comportamento ético em seus líderes, a criatividade é aprimorada por meio da aprendizagem social, ou seja, mediante as próprias ações. Os resultados revelam que o efeito indireto de liderança ética na criatividade é mais forte quando o clima propício à inovação na empresa é maior, desempenhando um papel crítico nessa interação (A. S.-Y. Chen \& Hou, 2016).

Percebe-se, dessa forma, a influência do líder sobre a criatividade dos funcionários e a importância para a organização da acertada seleção e delegação desse líder para a equipe de trabalho, uma das práticas essenciais da gestão de pessoas. Os métodos seletivos devem basear-se na seleção por competências, em que se avaliam as atitudes e os comportamentos anteriores, sobretudo na gestão de conflitos e táticas de gestão de conhecimento. Torna-se pertinente também o adequado treinamento desses líderes para engajamento satisfatório na organização (Bai et al., 2016; Huang, Krasikova \& Liu, 2016).

Num processo seletivo para novos funcionários, quando as empresas utilizam critérios claros na busca por características pessoais como capacidades criativas e inovadoras, estes tendem a gerar uma variedade de ideias e se comprometerem mais. Outro ponto a ser observado é a compatibilidade de atitudes e valores entre indivíduos e organização. Quando esses fatores estão alinhados entre os dois, funcionários tornam-se fontes de novas ideias no processo inovador da empresa (Ahmad \& Schroeder, 2003; C.-J. Chen \& Huang, 2009; Liu et al., 2007).

A criatividade é reforçada em empresas flexíveis e o acesso aos recursos necessários pode estimular a criatividade e a mudança (Amabile, 1997). Ambientes de trabalho inconsistentes, onde os valores e as práticas organizacionais são incoerentes, geram insegurança nos funcionários, impedindo-os de experimentar novas ideias e assumir riscos. Práticas claras e definidas de recursos humanos propiciam aos funcionários a sensação de estabilidade e segurança. Consequentemente, estes se envolvem e se dedicam, correndo riscos e aumentando sua produtividade e criatividade (Binyamin \& Carmeli, 2010). Logo, as organizações devem agir 
proativamente, eliminando os fatores que impedem a criatividade $\mathrm{e}$ implementando estímulos à criatividade (Amabile, 1997; Binyamin \& Carmeli, 2010; Liu et al., 2007).

A cultura da organização é outro fator relevante ao estímulo da criatividade (Y. Wang \& Wang, 2016). Sugere-se que os resultados criativos são consequência de ambientes de trabalho criativos, onde a cultura da empresa incentiva e recompensa a criatividade (Bai et al., 2016; K. K. Chen, 2012). A criatividade individual é facilitada nessas empresas, que são discretas em relação à forma de organização das tarefas de seus funcionários, e também quando há eficácia e qualidade de informação (Ahmad \& Schroeder, 2003).

Qualidades relacionadas ao ambiente de trabalho promovidas por meio das práticas de gestão de pessoas, como a liberdade sobre o controle e a realização da tarefa, ou seja, a autonomia operacional, podem estimular a criatividade. Autonomia é a extensão de liberdade que os funcionários possuem para levar uma ideia adiante e implementá-la (Amabile, 1997; Jambulingam et al., 2005). O auxílio na gestão de interesses dos funcionários de forma entusiasmada e corajosa é outra qualidade referente ao ambiente de trabalho. O acesso facilitado aos recursos e tempo necessários, o clima organizacional colaborativo e a aceitação de novas ideias, com valorização da inovação e aceitação de possíveis fracassos, além do desafio da tarefa e reconhecimento profissional são algumas das qualidades do ambiente de trabalho que promovem a criatividade (Amabile, 1988; Fischer et al., 2016). Assim, sugere-se que:

$H_{1}$ : A autonomia dada aos funcionários possui relação positiva e significante com a criatividade para inovação.

Outras práticas de recursos humanos, como a avaliação de desempenho corretamente aplicada e com feedback construtivo, promovem no funcionário o sentimento de compromisso e satisfação levando-o a trocar ideias e conhecimentos (Bai et al., 2016). A avaliação de desempenho possui duas finalidades, uma é a tarefa de administração, que determina atribuições, aumentos e promoções, e a outra é o desenvolvimento, que consiste em fornecer feedback, coaching, além de identificar os treinamentos e formações necessárias ao funcionário (Snell \& Dean, 1992). 
$H_{2}$ : A avaliação de desempenho possui relação positiva e significante com a criatividade para inovação.

O treinamento dissemina conhecimento, habilidade e capacidade dos funcionários para desempenhar eficazmente suas atividades. A gestão de carreira apoia os funcionários no alcance de suas metas e objetivos profissionais (Ahmad \& Schroeder, 2003). O sistema de recompensa motiva o funcionário, porque ele é reconhecido por seu desempenho, aumentando sua participação na contribuição de ideias inovadoras. Todas essas práticas aplicadas de forma eficaz refletem a maior inovação organizacional (Beugelsdijk, 2008; Liu et al., 2007; Mumford \& Gustafson, 1988; Tan \& Nasurdin, 2011).

$\mathrm{H}_{3}$ : Treinamento possui relação positiva e significante com a criatividade para inovação.

Os sistemas de recompensas têm o objetivo de incentivar os funcionários a colaborarem de maneira positiva para a empresa ao longo do tempo (Snell \& Dean, 1992). Alguns estudos, porém, apontam para a percepção negativa por parte dos funcionários em relação ao sistema de recompensas. Em alguns casos, funcionários o percebem como controlador ao invés de estimulador de desempenho. Este item deve ser analisado pelas empresas a fim de minimizar seu efeito negativo (Ahmad \& Schroeder, 2003).

H4: O sistema de recompensas possui relação positiva e significante com a criatividade para inovação.

Observa-se, ainda, que, quando os funcionários aprendem somente as tarefas de rotina de suas funções, não podem contribuir de maneira significativa para a organização além de suas atividades rotineiras. A participação refere-se ao grau de influência por parte dos funcionários nas decisões da empresa. Nas organizaçoes que incentivam essa prática, os indivíduos têm a oportunidade de tomar iniciativa, participar na tomada de decisões e compartilhar as opiniões sobre seus cargos e problemas relacionados ao trabalho. Participação leva os funcionários a agirem proativamente, influenciando na maneira como é realizado seu trabalho e formando um senso de domínio sobre suas tarefas. Permite aos funcionários 
a identificação e correção de problemas relacionados às tarefas, estimulandoos a inovar continuamente (Liu et al., 2007; Sarinf \& Dermott, 2003).

Um ambiente de trabalho que proporciona ações peculiares de empresas criativas, como a independência ou autoconfiança, provavelmente contribuirá para realização inovadora na organização. Dessa forma, sistemas organizacionais e educacionais que apoiam a autonomia e contribuem para a autoestima do funcionário podem impactar de forma positiva na inovação organizacional, pois auxiliam na inibição das causas de estresse no ambiente de trabalho (Beugelsdijk, 2008; Mumford \& Gustafson, 1988; Y. Wang \& Wang, 2016). Sugere-se que, quanto maior a autoestima de um indivíduo, maior será sua criatividade (Y. Wang \& Wang, 2016).

H5: A participação possui relação positiva e significante com a criatividade para inovação.

Diante desse contexto, percebe-se que as práticas de recursos humanos predispõem à inovação organizacional, tornando-se um recurso valioso para a empresa (Beugelsdijk, 2008). As empresas podem utilizar práticas de recursos humanos para estimular o comportamento e a perspectiva dos funcionários, agregando valor ao desenvolvimento de inovação. Por meio de eficazes práticas de recursos humanos, como recrutamento, treinamento, participação, avaliação de desempenho e compensação, pode-se ampliar a competência na introdução de novos produtos, serviços e sistema de gestão e alcançar melhores resultados de inovação (C.-J. Chen \& Huang, 2009; Mumford \& Gustafson, 1988; Tan \& Nasurdin, 2011).

\section{METODOLOGIA}

Esta pesquisa está nos moldes de um estudo quantitativo, descritivo. A pesquisa descritiva atende melhor à necessidade de resposta para este artigo, pois buscou-se descrever as práticas de recursos humanos que influenciam a criatividade dos funcionários. A pesquisa descritiva é estruturada de forma específica a medir características sobre a questão da pesquisa (Hair, Babin, Money \& Samouel, 2005). 
Os dados coletados são de corte transversal e foram coletados entre os meses de maio e junho de 2016. O corte transversal coleta dados uma única vez, dentro de um período estimado de tempo. As surveys de amostra são características dos estudos transversais, e é um procedimento de coleta de dados primários por meio de indivíduos (Hair et al., 2005). Para a pesquisa deste artigo, utilizaram-se surveys eletrônicas para coleta dos dados. 0 questionário autoadministrado, montado numa plataforma virtual e disponibilizado on-line, convidava todo e qualquer indivíduo que tivesse vínculo empregatício a participar. Questionários autoadministráveis são respondidos sem a presença do pesquisador, espera-se que o indivíduo tenha conhecimento e motivação para respondê-los sozinho (Hair et al., 2005).

O questionário quantitativo foi escolhido devido à praticidade e à relativa rapidez da coleta de dados. O questionário apresenta escalas de respostas de 1 a 5, do tipo Likert, em que 1 corresponde a discordo totalmente e 5 a concordo totalmente. A escala Likert mede o grau de concordância ou discordância dos respondentes referente a uma declaração (Cresswell, 2007). Antes da versão final do questionário ser administrada, foi realizado um pré-teste para verificar o entendimento e a clareza das questões.

A pesquisa final resultou em 129 respondentes, dos quais 10 foram desconsiderados após constatação de existência de outlier multivariado e missing values, restando assim, 119 questionários respondidos que puderam ser utilizados nesta pesquisa. Os outliers são observações atípicas, sendo notados como muito diferentes das demais observações (Corrar, Paulo \& Dias Filho, 2009). Foram desconsiderados também os missing values, compostos por alguns questionários que deixaram diversas opções sem resposta.

Para um breve perfil dos respondentes, foram levantados dados sobre as variáveis de controle (gênero, idade, função) por meio de cinco perguntas. Outras 33 questões foram subdivididas em uma variável dependente (criatividade) e cinco variáveis independentes (autonomia, treinamento, participação, sistema de recompensa e avaliação de desempenho).

Para a variável dependente criatividade, foram elaboradas seis questões baseadas na pesquisa de Amabile (1997), e buscou-se levantar dados referentes a fatores que estimulem e encorajem a criatividade 
individual dos funcionários. Para levantamento de dados sobre a variável independente autonomia, foram elaboradas quatro questões baseadas no artigo de Jambulingam et al. (2005), com as quais buscou-se verificar a autonomia dos funcionários nas empresas em que atuam. Para a variável treinamento, com base no artigo de Ahmad e Schroeder (2003), foram formuladas seis questões para medir a extensão de treinamento que os funcionários recebem para que possam realizar múltiplas tarefas.

A variável participação foi medida por meio de quatro questões baseadas na pesquisa de Sarinf e Dermott (2003), em que foi analisado o grau como o líder é percebido na interação com os membros da equipe. Nas seis questões que objetivam pesquisar a variável sistema de recompensa, também baseadas no artigo de Ahmad e Schroeder (2003), buscou-se averiguar se os planos de incentivos de grupo e planos de participação nos lucros são utilizados nas empresas. Por fim, para a variável avaliação de desempenho, buscou-se medir se há percepção positiva por parte dos funcionários, por meio de sete questões baseadas na pesquisa de Sarinf e Dermott (2003).

Em relação à análise de dados, efetuaram-se a análise de frequência do perfil dos respondentes e, em seguida, as análises relacionadas às questões das dimensões em estudo.

Após a análise descritiva, verificou-se a validade de conteúdo e unidimensionalidade das dimensões em estudo por meio da análise fatorial confirmatória. Para isso, analisaram-se as cargas fatoriais e a significância das questões dentro da dimensão à qual pertencem. Cargas padronizadas superiores a 0,6 e p-value inferior a 0,05 são desejadas (Hair et al., 2005). Retiraram-se da dimensão as questões que obtiveram carga e significância fora dos limites estabelecidos. Optou-se por manter pelo menos três questões em cada dimensão para viabilizar o uso da dimensão e possibilitar o cálculo dos índices de ajuste do modelo estrutural.

Foi verificada também a confiabilidade das dimensões por meio do alfa de Cronbach e Confiabilidade Composta, os quais devem ser superiores a 0,6. Por último, testou-se a relação entre as dimensões independentes e dependentes por meio da modelagem por equações estruturais. Para esta 
análise, foi utilizado o software estatístico AMOS, módulo do SPSS.

Verificou-se nessa análise a significância das relações.

\section{ANALISE DOS RESULTADOS}

A amostra pesquisada refere-se a 119 indivíduos empregados, e é formada predominantemente por funcionários do gênero feminino, representando 61,2\% (74) dos sujeitos identificados. Relativamente à faixa etária, observou-se que a maioria dos funcionários participantes tem idade entre 18 e 25 anos, representando o total de 60,3\% da amostra, seguidos por indivíduos com idade entre 26 e 32 anos (26,4\%). Ainda referentemente à faixa etária, o restante da amostra corresponde a 13,2\% distribuídos entre funcionários de até 54 anos.

Quanto ao nível de escolaridade, identificou-se que 57,9\% dos funcionários têm ensino superior completo; outros 30,6\%, o ensino médio; e 10,7\%, especialização. Apenas um indivíduo respondente tem mestrado. Analisando o cargo dos funcionários, identificou-se que quase metade da amostra $(47,1 \%)$ atua no setor administrativo. A outra parte está distribuída de ordem decrescente nos setores financeiro (20,7\%), comercial (14,9\%), produção $(9,1 \%)$, recursos humanos $(5,8 \%)$ e gerência $(2,5 \%)$.

Em seguida, verificou-se a unidimensionalidade dos construtos. $\mathrm{O}$ resultado do refinamento das dimensões e os coeficientes de confiabilidade são apresentados na Tabela 1. 
Tabela 1: Unidimensionalidade das dimensões

\begin{tabular}{|c|c|c|c|c|c|c|c|}
\hline Dimensão & Questão & $\begin{array}{l}\text { Carga Padroniza } \\
\text { da }\end{array}$ & S.E. & T-value & P-value & $\begin{array}{l}\text { Confiabilidade } \\
\text { Composta }\end{array}$ & $\begin{array}{l}\text { Alfa de } \\
\text { Cronbach }\end{array}$ \\
\hline \multirow{4}{*}{ Autonomia } & AUTO01 & 0,609 & & & & \multirow{3}{*}{0,67} & \multirow{3}{*}{0,64} \\
\hline & AUTO03 & 0,487 & 0,176 & 4,811 & $* * *$ & & \\
\hline & AUTO04 & 0,789 & 0,197 & 7,016 & $* * *$ & & \\
\hline & AVAL01 & 0,681 & & & & \multirow{6}{*}{0,83} & \multirow{6}{*}{0,83} \\
\hline \multirow{5}{*}{$\begin{array}{l}\text { Avaliação de } \\
\text { Desempenho }\end{array}$} & AVAL03 & 0,610 & 0,138 & 6,004 & *** & & \\
\hline & AVAL04 & 0,787 & 0,170 & 7,535 & $* * *$ & & \\
\hline & \begin{tabular}{|l|} 
AVAL05 \\
\end{tabular} & 0,662 & 0,158 & 6,474 & $* * *$ & & \\
\hline & AVAL06 & 0,637 & 0,139 & 6,252 & $* * *$ & & \\
\hline & \begin{tabular}{|l|} 
AVAL07 \\
\end{tabular} & 0,671 & 0,152 & 6,554 & $* * *$ & & \\
\hline \multirow{6}{*}{ Criatividade } & CRIAT01 & 0,788 & & & & \multirow{5}{*}{0,82} & \multirow{5}{*}{0,81} \\
\hline & CRIAT02 & 0,682 & 0,112 & 7,817 & $* * *$ & & \\
\hline & CRIAT03 & 0,653 & 0,099 & 7,421 & $* * *$ & & \\
\hline & CRIAT04 & 0,676 & 0,116 & 7,729 & $* * *$ & & \\
\hline & CRIAT05 & 0,619 & 0,107 & 6,971 & $* * *$ & & \\
\hline & PART01 & 0,635 & & & & \multirow{3}{*}{0,71} & \multirow{3}{*}{0,71} \\
\hline \multirow[t]{2}{*}{ Participação } & \begin{tabular}{|l|} 
PART02 \\
\end{tabular} & 0,644 & 0,166 & 5,841 & $* * *$ & & \\
\hline & PART03 & 0,730 & 0,187 & 6,427 & $* * *$ & & \\
\hline \multirow{4}{*}{$\begin{array}{l}\text { Sistema de } \\
\text { Recompensa }\end{array}$} & SISTREC01 & 0,774 & & & & \multirow{4}{*}{0,92} & \multirow{4}{*}{0,92} \\
\hline & SISTREC02 & 0,899 & 0,102 & 10,877 & $* * *$ & & \\
\hline & \begin{tabular}{|l|} 
SISTREC03 \\
\end{tabular} & 0,866 & 0,100 & 10,372 & $* * *$ & & \\
\hline & SISTREC04 & 0,905 & 0,100 & 10,967 & $* * *$ & & \\
\hline \multirow{3}{*}{ Treinamento } & TREIN01 & 0,685 & & & & \multirow{3}{*}{0,78} & \multirow{3}{*}{0,77} \\
\hline & TREIN02 & 0,774 & 0,151 & 6,601 & $* * *$ & & \\
\hline & TREIN04 & 0,735 & 0,161 & 6,428 & $* * *$ & & \\
\hline
\end{tabular}

Fonte: dados da pesquisa

Verifica-se, na Tabela 1, que foram excluídas três questões no total, em dimensões diferentes: autonomia, avaliação de desempenho e 
treinamento. As demais questões apresentaram significância no nível de 0,01, e as dimensões apresentaram níveis aceitáveis de confiabilidade.

Em seguida, foi realizada a análise da validade discriminante entre dimensões, a qual é apresentada na Tabela 2.

Tabela 2: Validade discriminante das dimensões

\begin{tabular}{|c|c|c|c|c|c|c|c|}
\hline \multirow{2}{*}{\multicolumn{2}{|c|}{ Avaliação em Pares }} & \multicolumn{2}{|c|}{$\begin{array}{l}\text { Com } \\
\text { restrição }\end{array}$} & \multicolumn{2}{|c|}{$\begin{array}{l}\text { Sem } \\
\text { restrição }\end{array}$} & \multirow[t]{2}{*}{$\Delta x^{2}$} & \multirow[t]{2}{*}{ sig } \\
\hline & & \multirow{2}{*}{$\begin{array}{l}x^{2} \\
61,550\end{array}$} & \multirow{2}{*}{\begin{tabular}{|l|} 
df \\
27 \\
\end{tabular}} & \multirow{2}{*}{\begin{tabular}{|l}
$x^{2}$ \\
54,66
\end{tabular}} & \multirow{2}{*}{\begin{tabular}{|l|} 
df \\
26
\end{tabular}} & & \\
\hline Autonomia & Avaliação de Desempenho & & & & & 6,890 & $\overline{0,009}$ \\
\hline & Criatividade & 43,474 & 20 & 38,856 & 19 & 4,618 & 0,032 \\
\hline & Participação & 22,395 & 9 & 12,041 & 8 & 10,354 & 0,001 \\
\hline & Sistema de Recompensa & 15,459 & 14 & 6,385 & 13 & 9,074 & 0,003 \\
\hline & Treinamento & 26,526 & 9 & 14,476 & 8 & 12,05 & 0,001 \\
\hline \multirow{4}{*}{$\begin{array}{l}\text { Avaliação de } \\
\text { Desempenho }\end{array}$} & Criatividade & 90,860 & 44 & 86,663 & 43 & 4,197 & 0,040 \\
\hline & Participação & 71,825 & 27 & 60,523 & 26 & 11,302 & 0,001 \\
\hline & Sistema de Recompensa & 66,696 & 35 & 63,703 & 34 & 2,993 & 0,084 \\
\hline & Treinamento & 51,865 & 27 & 43,666 & 26 & 8,199 & 0,004 \\
\hline \multirow[t]{3}{*}{ Criatividade } & Participação & 46,396 & 20 & 39,691 & 19 & 6,705 & 0,010 \\
\hline & Sistema de Recompensa & 45,306 & 27 & 40,091 & 26 & 5,215 & 0,027 \\
\hline & Treinamento & 54,558 & 20 & 47,539 & 19 & 7,019 & 0,008 \\
\hline \multirow[t]{2}{*}{ Participação } & Sistema de Recompensa & 19,093 & 14 & 9,42 & 13 & 9,673 & 0,002 \\
\hline & Treinamento & 35,869 & 9 & 25,909 & 8 & 9,96 & 0,002 \\
\hline \multicolumn{8}{|l|}{ Sistema de } \\
\hline Recompensa & Treinamento & 27,194 & 14 & 16,996 & 13 & 10,198 & 0,001 \\
\hline
\end{tabular}

Fonte: dados da pesquisa 
De acordo com a Tabela 2, todas as dimensões são diferentes no nível de 0,05 , o que significa a não existência de colinearidade entre as variáveis independentes em estudo.

Verificada a validade de conteúdo, unidimensionalidade e discriminante, procedeu-se ao teste do modelo estrutural, o qual considera as práticas de recursos humanos que podem influenciar na criatividade dos funcionários. O teste do modelo estrutural é apresentado na Tabela 3.

Tabela 3: Teste do Modelo Estrutural

\begin{tabular}{l|l|l|l|l|l|l}
\hline $\begin{array}{l}\text { Variáveis } \\
\text { Independentes }\end{array}$ & $\begin{array}{l}\text { Variável } \\
\text { Dependente }\end{array}$ & Coeficiente & $\begin{array}{l}\text { Erro } \\
\text { Padrão }\end{array}$ & C.R. & $\begin{array}{l}\text { P- } \\
\text { value }\end{array}$ & $\mathbf{R}^{\mathbf{2}}$ \\
\hline Autonomia & & 0,717 & 0,150 & 4,577 & $* * *$ & \\
\hline $\begin{array}{c}\text { Avaliação de } \\
\text { Desempenho }\end{array}$ & \multirow{2}{*}{ Criatividade } & 0,238 & 0,089 & 2,298 & 0,022 & \multirow{2}{*}{0,912} \\
\cline { 4 - 7 } & & & 0,070 & 4,417 & $* * *$ & \\
\hline Participação & & 0,161 & 0,064 & 1,732 & 0,083 & \\
\hline Treinamento & & 0,311 & 0,054 & 3,387 & $* * *$ & \\
\hline Recompensa & & & & & & \\
\hline
\end{tabular}

Fonte: dados da pesquisa

Os dados apresentados na Tabela 3 sugerem que autonomia é a variável independente que exerce maior influência $(0,717)$ na criatividade dos funcionários, com cerca de $51 \%$ de explicação. Essa influência pode ser explicada pela liberdade que os funcionários têm para executar as atividades e tomar decisões pertinentes a seu trabalho sem a interferência dos superiores. Assim, como forma de melhorar o desempenho da área em que atuam, os funcionários buscam encontrar as melhores soluções para a realização das atividades, exercendo a criatividade (Amabile, 1988, 2013; Amabile et al., 1996).

A avaliação de desempenho também obteve influência positiva e significante sobre a criatividade $(0,467)$, com um grau de explicação de $21,8 \%$. A avaliação de desempenho oferece informações aos funcionários que são relevantes para o aprimoramento da carreira, gerando satisfação e 
comprometimento com a organização à qual estão vinculados. Além disso, a avaliação de desempenho pode identificar potencias fragilidades individuais que podem ser amenizadas por meio de treinamentos, troca de ideias e conhecimentos, influenciando de maneira positiva a criatividade (Bai et al., 2016; Snell e Dean, 1992).

A variável recompensa, por sua vez, também obteve influência positiva e significante sobre a criatividade dos funcionários, com um grau de explicação de 9,6\%. Assim, verificou-se que os planos de incentivos são percebidos de forma positiva pelos funcionários, que constatam o reconhecimento por parte da organização pelo desempenho que apresentaram. Dessa forma, os funcionários se sentem mais motivados para contribuir com ideias inovadoras, melhorando o desempenho organizacional (Beugelsdijk, 2008; Liu et al., 2007; Mumford \& Gustafson, 1988; Tan \& Nasurdin, 2011).

Dentre as variáveis que obtiveram influência significativa sobre a criatividade, segue a participação dos funcionários nas decisões das empresas. Essa influência é de aproximadamente 0,238, o que corresponde a um poder de explicação de 5,6\%. A participação dos funcionários os motiva a agir de forma proativa, alterando as formas de execução do trabalho e ganhando consciência e maior conhecimento sobre o trabalho. Essas ações são descritas por Sarinf e Dermontt (2003) e Liu et al. (2007) como estímulos a criatividade e inovação.

Por último, embora o treinamento tenha uma influência positiva sobre a criatividade, tal influência não apresenta resultados estatisticamente significantes ( $p$-value $=0,083$ ). Dessa forma, os achados deste estudo não corroboram estudos anteriores que estabeleceram relações significativas entre as variáveis. Assim, embora o treinamento promova 0 compartilhamento de conhecimento entre os funcionários no exercício das funções (Ahmad \& Schroeder, 2003), na amostra estudada, o treinamento não obteve relevância dada a expressividade das demais variáveis como autonomia, avaliação de desempenho e recompensa.

Em resumo, no Quadro 1, é apresentado, por meio do modelo estrutural, o resultado do teste das hipóteses, descritas anteriormente. 


\begin{tabular}{|l|l|l|}
\hline Hipótese & Descrição da Hipótese & Resultado \\
\hline $\mathrm{H}_{1}$ & $\begin{array}{l}\text { A autonomia dada aos funcionários possui relação positiva } \\
\text { e significante com a criatividade para inovação. }\end{array}$ & Não rejeitada \\
\hline $\mathrm{H}_{2}$ & $\begin{array}{l}\text { A avaliação de desempenho possui relação positiva e } \\
\text { significante com a criatividade para inovação. }\end{array}$ & Não rejeitada \\
\hline $\mathrm{H}_{3}$ & $\begin{array}{l}\text { Treinamento possui relação positiva e significante com a } \\
\text { criatividade para inovação. }\end{array}$ & Rejeitada \\
\hline $\mathrm{H}_{4}$ & $\begin{array}{l}\text { O sistema de recompensas possui relação positiva e } \\
\text { significante com a criatividade para inovação. }\end{array}$ & Não rejeitada \\
\hline $\mathrm{H}_{5}$ & $\begin{array}{l}\text { A participação possui relação positiva e significante com a } \\
\text { criatividade para inovação. }\end{array}$ & Não rejeitada \\
\hline
\end{tabular}

\section{Quadro 1: Resultado do teste de hipóteses}

Fonte: dados da pesquisa

Conforme o Quadro 1, apenas uma das hipóteses foi rejeitada. As hipóteses não rejeitadas, que representam a influência da autonomia, avaliação de desempenho, sistemas de recompensa e participação sobre a criatividade, explicam este fenômeno em 91,2\%.

Ressalta-se que nessas análises não foram confrontados os resultados obtidos nesta pesquisa com resultados de pesquisas anteriores, uma vez que não se encontraram estudos que tivessem o mesmo foco ou que testassem essas relações empriricamente. Assim, as análises foram baseadas em lógicas estabelecidas a partir do entendimento da literatura sobre práticas de gestão de pessoas e criatividade.

\section{CONSIDERAÇÕES FINAIS}

Neste artigo, o objetivo foi descrever as práticas de recursos humanos que estimulam ou influenciam a criatividade dos funcionários. Para isso foram coletados dados com indivíduos que possuíam vínculo empregatício. Os dados foram analisados por meio da modelagem por equações estruturais, que testa a relação entre as práticas de recursos humanos e a criatividade dos funcionários.

Os resultados apontaram que a autonomia, como liberdade para execução das tarefas e tomada de decisão relativa à função, assim como o 
diálogo da chefia com os funcionários sobre o desempenho do trabalhador, são as duas práticas de recursos humanos mais influentes na criatividade dos funcionários. Essas práticas refletem um ambiente de trabalho em que os funcionários se sentem motivados não só por tomarem decisões referentes a sua função, mas também por ter ligação direta com a chefia por meio de conversas que expressam o desempenho do trabalhador. Essas duas práticas, em conjunto, podem explicar $72,8 \%$ da criatividade dos funcionários.

Adicionalmente, a existência de sistemas de recompensa e o sentimento de participação no processo de tomada de decisão da estratégia da empresa podem estimular a geração de ideias inovadoras e contribuir para a solução dos problemas organizacionais. Embora em menor expressão, essas outras duas práticas de recursos humanos podem explicar a criatividade dos funcionários em cerca de $15,1 \%$. Isso significa que o comprometimento dos funcionários na geração de ideias que possam contribuir para a inovação organizacional não é motivado por fatores financeiros, mas, sim, por ações que proporcionem o senso de pertencimento à empresa e de reconhecimento de seu trabalho.

Os achados deste estudo corroboram estudos anteriores, com exceção do investimento em treinamento, cuja influência sobre a criatividade não foi verificada. Esse resultado pode ser explicado pelo foco do treinamento para capacitação na execução de tarefas e não em formas de geração de ideias.

Em termos acadêmicos, neste estudo se oferece um modelo sintético que analisa as principais práticas de gestão de pessoas que influenciam a criatividade, uma vez que não se encontraram na literatura existente modelos que explicassem a participação dessas práticas no processo de geração de ideias inovadoras. Em termos empíricos, empresas podem beneficiar-se do resultado desta pesquisa fomentando ações que estimulem funcionários no desenvolvimento de equipes autogerenciáveis e empowerment e fornecendo informação clara e concisa sobre o desempenho individual e organizacional.

As limitações deste estudo incorrem sobre a não especificação de uma indústria para estudo. Esta pesquisa foi realizada de forma geral, abrangendo trabalhadores das mais diversas empresas e segmentos. Outra limitação está relacionada à percepção dos funcionários sobre as práticas de gestão de 
pessoas, pois eles podem não perceber a prática de gestão de pessoas adotada pela organização.

Dessa forma, sugere-se em pesquisa futura um comparativo entre funcionários de empresas de segmentos menos inovadores com aqueles que demandam maior inovação. Assim, será possível descrever os fatores que influenciam a criatividade em ambientes complexos e bem definidos.

\section{REFERÊNCIAS}

Afacan Fındıklı, M., Yozgat, U., \& Rofcanin, Y. (2015). Examining Organizational Innovation and Knowledge Management Capacity The Central Role of Strategic Human Resources Practices (SHRPs). Procedia Social and Behavioral Sciences, 181, 377-387. doi: 10.1016/j.sbspro.2015.04.900

Agnoli, S., \& Corazza, G. E. (2015). TRIZ as Seen through the DIMAI Creative Thinking Model. Procedia Engineering, 131, 807-815. doi: 10.1016/j.proeng.2015.12.382

Ahmad, S., \& Schroeder, R. G. (2003). The impact of human resource management practices on operational performance: recognizing country and industry differences. Journal of Operations Management, 21(1), 1943. doi: $10.1016 / \mathrm{S} 0272-6963(02) 00056-6$

Amabile, T. M. (1988). A model of creativity and innovation in organizations. Research in Organizational Behavior, 10, 123-167.

Amabile, T. M. (1997). Motivating creativity in organizations: on doing what you love and loving what you do. California Management Review, 40, 3958.

Amabile, T. M. (1998). How to kill creativity. Harvard Business Review, 76(5), 77-87.

Amabile, T. M. (2013). Componential theory of creativity. In E. H. Kessler (Ed.), Encyclopedia of management theory. London: Sage.

Amabile, T. M., Barsade, S. G., Mueller, J. S., \& Staw, B. M. (2005). Affect and creativity at work. Administrative Science Quarterly, 50(3), 367-403.

Amabile, T. M., Conti, R., Coon, H., Lazenby, J., \& Herron, M. (1996). Assessing the work environment for creativity. Academy of Management Journal, 39, 1154-1184.

Bai, Y., Lin, L., \& Li, P. P. (2016). How to enable employee creativity in a team context: a cross-level mediating process of transformational leadership. Journal of Business Research, 69(9), 3240-3250. doi: 10.1016/j.jbusres.2016.02.025 
Beugelsdijk, S. (2008). Strategic human resource practices and product innovation. Organization Studies, 29, 821-847.

Binyamin, G., \& Carmeli, A. (2010). Does structuring of human resource management processes enhance employee creativity? The mediating role of psychological availability. Human Resource Management, 49(6), 9991024. doi: $10.1002 / \mathrm{hrm} .20397$

Borghini, S. (2005). Organizational creativity: breaking equilibrium and order to innovate. Journal of Knowledge Management, 9(4), 19-33. doi: doi: $10.1108 / 13673270510610305$

Chen, A. S.-Y., \& Hou, Y.-H. (2016). The effects of ethical leadership, voice behavior and climates for innovation on creativity: A moderated mediation examination. The Leadership Quarterly, 27(1), 1-13. doi: 10.1016/j.leaqua.2015.10.007

Chen, C.-J., \& Huang, J.-W. (2009). Strategic human resource practices and innovation performance - The mediating role of knowledge management capacity. Journal of Business Research, 62(1), 104-114. doi: 10.1016/j.jbusres.2007.11.016

Chen, K. K. (2012). Organizing creativity: enabling creative output, process, and organizing practices. Sociology Compass, 6(8), 624-643. doi: 10.1111/j.1751-9020.2012.00480.x

Corrar, L. J., Paulo, E., \& Dias Filho, J. M. D. (2009). Análise multivariada para os cursos de administração, ciências contábeis e economia. São Paulo: Atlas.

Cresswell, J. W. (2007). Projeto de pesquisa: métodos qualitativo, quantitativo e misto (2a ed.). Porto Alegre: Artmed.

Fischer, S., Oget, D., \& Cavallucci, D. (2016). The evaluation of creativity from the perspective of subject matter and training in higher education: issues, constraints and limitations. Thinking Skills and Creativity, 19, 123135. doi: $10.1016 /$ j.tsc.2015.10.002

Gabriel, A. S., Cheshin, A., Moran, C. M., \& van Kleef, G. A. (2016). Enhancing emotional performance and customer service through human resources practices: A systems perspective. Human Resource Management Review, 26(1), 14-24. doi: 10.1016/j.hrmr.2015.09.003

Hair, J. F. J., Babin, B., Money, A. H., \& Samouel, P. (2005). Fundamentos de métodos de pesquisa em administração. Porto Alegre: Bookman.

Hiltrop, J.-M. (1999). The quest for the best: human resource practices to attract and retain talent. European Management Journal, 17(4), 422-430. doi: $10.1016 / S 0263-2373(99) 00022-5$

Huang, L., Krasikova, D. V., \& Liu, D. (2016). I can do it, so can you: the role of leader creative self-efficacy in facilitating follower creativity. 
Organizational Behavior and Human Decision Processes, 132, 49-62. doi: 10.1016/j.obhdp.2015.12.002

Jambulingam, T., Kathuria, R., \& Doucette, W. R. (2005). Entrepreneurial orientation as a basis for classification within a service industry: the case of retail pharmacy industry. Journal of Operations Management, 23(1), 23-42. doi: 10.1016/j.jom.2004.09.003

Liu, Y., Combs, J. G., Ketchen Jr, D. J., \& Ireland, R. D. (2007). The value of human resource management for organizational performance. Business Horizons, 50(6), 503-511. doi: 10.1016/j.bushor.2007.07.002

Mumford, M. D., \& Gustafson, S. B. (1988). Creativity syndrome: integration, application, and innovation. Psychological Bulletin, 103, 27-43.

Nadarajah, S., Kadiresan, V., Kumar, R., Kamil, N. N. A., \& Yusoff, Y. M. (2012). The relationship of HR practices and job performance of academicians towards career development in Malaysian private higher institutions. Procedia - Social and Behavioral Sciences, 57, 102-118. doi: 10.1016/j.sbspro.2012.09.1163

Nayak, R. C., Noida, G., \& Agarwal, R. (2011). A model of creativity and innovation in organizations. International Journal of Transformations in Business Management - IJTBM, 1(1), 1-8.

Paşaoğlu, D., \& Tonus, H. Z. (2014). Strategic importance of human resource practices on job satisfaction in private hospitals. Procedia - Social and Behavioral Sciences, 150, 394-403. doi: 10.1016/j.sbspro.2014.09.035

Rogg, K. L., Schmidt, D. B., Shull, C., \& Schmitt, N. (2001). Human resource practices, organizational climate, and customer satisfaction. Journal of Management, 27(4), 431-449. doi: 10.1016/S0149-2063(01)00102-7

Sarinf, S., \& Dermott, C. M. (2003). The effect of team leader characteristics on learning, knowledge application, and performance of cross-functional new product development teams. Decision Sciences, 34, 707-739.

Slavich, B., Cappetta, R., \& Giangreco, A. (2014). Exploring the link between human resource practices and turnover in multi-brand companies: the role of brand units' images. European Management Journal, 32(2), 177-189. doi: $10.1016 /$ j.emj.2013.06.001

Snell, S. A., \& Dean, J. W. J. (1992). Integrated manufacturing and human resource management a human capital perspective. Academy of Managemenl Journal, 35, 467-504.

Tan, C. L., \& Nasurdin, A. M. (2011). Human resource management practices and organizational innovation: assessing the mediating role of knowledge management effectiveness. The Electronic Journal of Knowledge Management, 9(2), 155-167. 
Udwadia, F. E. (1990). Creativity and innovation in organizations: two models and managerial implications. Technological Forecasting and Social Change, 38(1), 65-80. doi: 10.1016/0040-1625(90)90018-Q

Wang, X.-H., Kim, T.-Y., \& Lee, D.-R. (2016). Cognitive diversity and team creativity: effects of team intrinsic motivation and transformational leadership. Journal of Business Research. doi: 10.1016/j.jbusres.2016.02.026

Wang, Y., \& Wang, L. (2016). Self-construal and creativity: the moderator effect of self-esteem. Personality and Individual Differences, 99, 184-189. doi: $10.1016 /$ j.paid.2016.04.086

Werbel, J., \& Balkin, D. B. (2010). Are human resource practices linked to employee misconduct?: A rational choice perspective. Human Resource Management Review, 20(4), 317-326. doi: 10.1016/j.hrmr.2009.10.002

Zehir, C., Gurol, Y., Karaboga, T., \& Kole, M. (2016). Strategic human resource management and firm performance: the mediating role of entrepreneurial orientation. Procedia - Social and Behavioral Sciences, 235, 372-381. doi: 10.1016/j.sbspro.2016.11.045 